\title{
Equally Flexible and Optimal Response Bias in Older Compared to Younger Adults:
}

\section{Supplemental Materials}

Supplementary to the analysis of the original experimental data described in the main report, the following materials are offered as background information and ancillary analyses. Some further methodological details are also reported here. In the sections listed below, table numbers, figures and references refer to these supplemental materials unless otherwise indicated.

\section{Table of contents}

1. Method

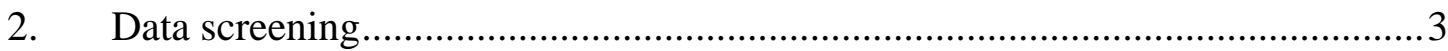

3. Linear Mixed Effects Modelling of Response Times and Error Rates ...............4

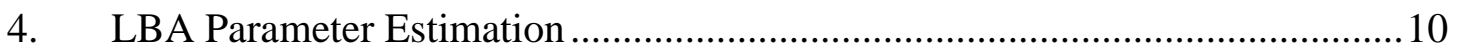

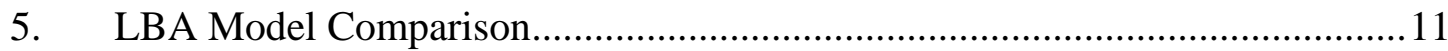

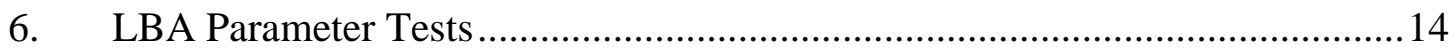

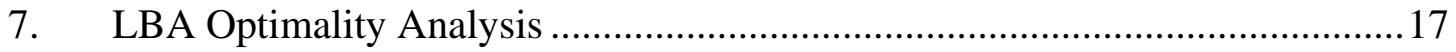

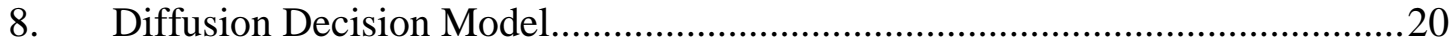

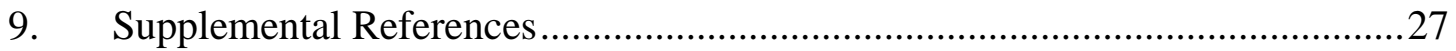




\section{Method}

In addition to the methodological details about our experiment in the main report, we provide the following details concerning location, dates, equipment and randomisation procedures.

The experiment was administered in the Tasmanian Cognition Laboratory of the University of Tasmania across April 2017 to February 2018 during standard working hours under normal ambient illumination. It was run on desktop computers equipped with $3.30 \mathrm{GHz}$ Intel i5-6600 processors, 16 GB RAM, and a Windows 7 Enterprise operating system configured to minimise internal task-switching. It was written in JavaScript and run locally using a Firefox (v. 46.0.1) browser. A single experimenter (AR or RG, both Ph.D. candidates) ran one or two participants per session (40\% of the younger participants, in addition to 12 software-development pilot runs, by AR; otherwise by RG). These experimenters were also responsible for recruiting participants. The interval between the two sessions ranged from 1 to 14 days (mean $=5, S D=3$ ).

For the random assignment of trials to the conditions of target colour, discrimination difficulty and cue-congruence, sequences encoding these conditions (to the specifications given in the main report) were pseudo-randomly generated (by AR) using the $\mathrm{R}$ implementation of the Mersenne-Twister algorithm. The sequences were pseudo-randomly distributed among participants, with about $30 \%$ being reused between participants for convenience. No sequences showed any deviation from randomness (by, e.g., runs tests) other than what could be attributable to chance, with respect to the different biasing conditions. 


\section{Data screening}

The experiment produced 38,400 trials over all conditions, with 19,200 trials for each age-group, with equal separations at the levels of bias-type and discrimination difficulty, and with separations per cue-target congruence and target colour according to the level of biastype. The trial-level data are available on the Open Science Framework (https://osf.io/9hwu2/).

Prior to analysis, data were screened for "too fast" responses, and response timeouts. Inspection of per-participant RT distributions indicated that a minimum RT cut-off of $0.2 \mathrm{~s}$ was sufficient to exclude implausibly fast responses among both older and younger participants (7 and 24 trials, respectively). The rarity of these fast responses indicated that fast guessing was generally absent from performance. Older participants produced more response timeouts than younger participants (66 and 22 trials, respectively); a preliminary indication of greater caution with age in the data (as in, e.g., Silverman, 1963). Also, there were about twice as many response timeouts under trial-wise (58) than block-wise (30) biasing; a preliminary indication — consistent with our procedural intentions - that performance under trial-wise biasing was more difficult than under block-wise. Nevertheless, the rarity of response timeouts indicated that participants were well motivated to perform according to task instructions. Altogether, these screening procedures excluded $0.3 \%$ of trials. 


\section{Linear Mixed Effects Modelling of Response Times and Error Rates}

For analysis of effects of the factors in the study design on the standard measures of performance — response time (RT and error rate (ER) — mixed-effects models (per the lme4 R package, v. 1.1-19, Bates, Mächler, Bolker, \& Walker, 2015) were used to account for bysubject random intercepts and any potential by-subject random effects of the factors (Baayen, Davidson, \& Bates, 2008; Baayen \& Milin, 2010). The RTs were log-transformed and analysed assuming a Gaussian distribution, and ERs were analysed assuming a binomial distribution with a probit link function. Log-transformed RTs better satisfied the assumptions of regression modelling (as per examination of residuals) than inverse-Gaussian transformed RTs, and the raw RTs themselves.

Analysis commenced with a model that allowed for all possible interactive and main effects of the fixed factors, and by-subject random intercepts (see Table 1, "Full model"). Simpler fixed effects structures, and more complex random effects structures, were explored through forward and backward fits of multiple alternative factor partitions, with model comparison and selection based on likelihood ratio tests (aiming for reductions in complexity-penalising goodness-of-fit measures, viz., AIC and BIC) and ANOVA-equivalent tests (with Kenward-Roger $d f$ estimation per the lmerTest R package, v. 3.0-1, Kuznetsova, Brockhoff, \& Christensen, 2017). This procedure was not as extensively possible for models of error rates as they suffered multiple convergence issues (see Eager \& Roy, 2017).

Common to all selected models, there were main effects of age-group, congruence and difficulty but not bias-type and target colour; and bias-type was effective only in interaction with the effect of congruence. Accounting for by-subject random effects of biastype and target colour improved estimation of RTs; random effects were not reliably estimable for ERs. Bias-type accounted for the largest proportion of subject-wise variance. 
These random effects are consistent with indications of individual differences in postparticipation reports concerning cue use (e.g., from some participants describing a strategy of testing cue validity, to others describing the cues as distracting) and the relative "salience" of the target colours. The overall proportion of variance accounted for by these models was at a typically moderate level for RTs but quite small for ERs. Eliminating observations associated with large residuals (Baayen \& Milin, 2010; Davies, Arnell, Birchenough, Grimmond, \& Houlson, 2017) had no impact on satisfying modelling assumptions and on the pattern of effects. 
Table 1. Full and Selected Model Structures and Fit Measures.

\begin{tabular}{|c|c|c|c|c|c|c|c|c|c|}
\hline \multirow[t]{2}{*}{$\mathrm{DV}$} & \multicolumn{3}{|c|}{ Full model } & \multirow[b]{2}{*}{$R^{2}$} & \multicolumn{5}{|c|}{ Selected model } \\
\hline & $d f$ & AIC & $\mathrm{BIC}$ & & $d f$ & AIC & $\mathrm{BIC}$ & $R^{2}$ & Formula \\
\hline Correct RTs & 34 & 1183 & 1468 & 0.422 & 30 & -1457 & -1205 & 0.467 & $\mathrm{C}: \mathrm{B}+\mathrm{D} *(\mathrm{~A}+\mathrm{C})+(\mathrm{C} * \mathrm{~B}+\mathrm{D}+\mathrm{T} \mid \mathrm{s})$ \\
\hline Error RTs & 34 & 2669 & 2895 & 0.491 & 17 & 2112 & 2225 & 0.535 & $\mathrm{C}: \mathrm{B}+\mathrm{C}+\mathrm{A}+\mathrm{D}+(\mathrm{C}+\mathrm{B}+\mathrm{T} \mid \mathrm{s})$ \\
\hline Error rates & 33 & 29952 & 30243 & 0.152 & 17 & 29937 & 30082 & 0.151 & $\mathrm{C} * \mathrm{~B} * \mathrm{~A} * \mathrm{D}+(1 \mid \mathrm{s})$ \\
\hline
\end{tabular}

Note. $R^{2}=R_{\mathrm{LR}}{ }^{2}$ for RTs and $R_{\mathrm{GLLM}}{ }^{2}$ for error rates (per the MuMIn R package, v. 1.42.1;

Bartoń, 2018). Formulas are in the standard computational format with first-letter abbreviations of the factors defined in the Design section: $\mathrm{C}=$ cue-target congruence; $\mathrm{B}=$ bias-type; $\mathrm{D}=$ discrimination difficulty; $\mathrm{A}=$ age-group; $\mathrm{T}=\operatorname{target}$ colour; $\mathrm{s}=$ participant. The full model had the formula $C * B * A * D * T+(1 \mid s)$. 
The following details the between-group and then within-group effects that, following model comparison, were confirmed to be substantial and significant (with $\alpha=.05$ ). We report unstandardized effect sizes as the differences between the relevant parameter estimates while averaging over parameters not included in the comparison. Standardized effect sizes are not reported given that there are no standard procedures for their estimation from mixed models (e.g., Singmann \& Kellen, in press). Descriptives reported in the following text are marginal means (and 95\% $t$-distribution confidence intervals), as estimated from the mixed effects models and back-transformed from log/probit scales.

Age-related slowing and accuracy. Responses were much slower for older than younger participants- both when correct $[0.778 \mathrm{~s}(0.730 \mathrm{~s}, 0.829 \mathrm{~s})$ and $0.577 \mathrm{~s}(0.541 \mathrm{~s}$, $0.615 \mathrm{~s})$, respectively] and incorrect $[0.853 \mathrm{~s}(0.780 \mathrm{~s}, 0.931 \mathrm{~s})$ and $0.553 \mathrm{~s}(0.506 \mathrm{~s}, 0.604 \mathrm{~s})$, respectively]. As these descriptives indicate, errors were slower than correct responses among older participants, whereas younger participants tended to make faster error responses than correct responses. Error rates were also slightly lower for older $[13.4 \%(11.4 \%, 15.7 \%)]$ than younger participants $[16.3 \%(14.0 \%, 18.8 \%)]$. Across both age-groups, there were slower correct and error responses, and more errors, for hard than for easy discriminations. Between age groups, the difficulty cost on correct RTs tended to be larger for older $(0.055 \mathrm{~s})$ than younger participants (0.026s). Similarly, the cost on accuracy by discrimination difficulty was slightly larger for older (13.8\%) than younger participants (12.9\%), pairwise comparisons indicating that older participants had fewer errors than younger participants more reliably on easy trials $(Z=2.44)$ than on hard trials $(Z=0.93)$. This replicates the common observation that the cost of difficulty increases with age. 
Biasing effects. Correct responses were faster, error responses were slower, and accuracy was greater for the more likely (cue-congruent) than less likely (cue-incongruent) target colours. Although there was no statistically significant main effect of bias-type on any measure, the block-wise biasing effect was, as predicted, larger on all measures than the trialwise biasing effect — correct responses were faster (on congruent compared to incongruent trials) by $0.06 \mathrm{~s}$ and $0.04 \mathrm{~s}$ under block-wise and trial-wise biasing, respectively; error responses were slower by $0.1 \mathrm{~s}$ and $0.045 \mathrm{~s}$, respectively; and error rates were reduced by $12 \%$ and $6 \%$, respectively. These differences in the biasing effect between bias-types were produced by differences on cue-congruent trials when responses were correct, and by differences on cue-incongruent trial when responses were incorrect. Specifically, for correct RTs, responses on cue-congruent trials were $0.023 \mathrm{~s}$ faster under block-wise than trial-wise biasing, but cue-incongruent responses differed by only $0.001 \mathrm{~s}$ between bias-types. For error RTs, conversely, responses on cue-incongruent trials were almost 0.050 s faster under blockwise than trial-wise biasing, whereas responses on cue-congruent trials were $0.011 \mathrm{~s}$ slower for block-wise than for trial-wise bias. In relation to discrimination difficulty, the effect of cue-congruence on correct RTs was slightly greater (by $0.012 \mathrm{~s}$ ) on hard relative to easy trials across bias-types.

Age and biasing effects. Older participants acquired at least as much advantage from the bias cues as did younger participants. Specifically, there was no significant interaction of age with the effects of cue-congruence and/or bias-type on correct or error RTs. In fact, for correct RTs, the biasing effect was, on average, slightly larger for older than younger participants, both under block-wise biasing (by $0.07 \mathrm{~s}$ and $0.055 \mathrm{~s}$, respectively) and under trial-wise biasing (by $0.05 \mathrm{~s}$ and $0.03 \mathrm{~s}$, respectively). With respect to the RT distributions (see Figures 1 and 2 in the main report), the biasing effect was generally stronger for faster 
responses; and, among their fastest $10 \%$ of responses, older and younger participants showed substantially equivalent biasing effects. However, the biasing effect remained strong throughout the RT distribution for older participants (e.g., 0.066s and 0.048s for the lowest and highest $10 \%$ of correct RTs, respectively, on easy block-biased trials; see Figure 1 in the main report, top left panel) but not for younger participants $(0.069 \mathrm{~s}$ and $0.009 \mathrm{~s}$, respectively; see Figure 2 in the main report, top left panel). For ERs, the biasing effect was significantly greater among older than younger participants, subserved by a slightly larger effect among older than younger participants under block-wise biasing (12.6\% and $11.8 \%$, respectively), with little age difference under trial-wise biasing (6.5\% and $6.3 \%$, respectively).

The data were also examined for block-wise trends and sequential effects. For brevity, only the following key findings are presented here. As for block-wise trends, RTs declined over blocks much more among older than younger participants, especially under block-wise biasing; younger participants showed no difference in trends between bias-types. Accuracy was stable under all conditions across the sessions. For correct RTs, biasing effects occurred within the earliest blocks under both bias-types, and the block-wise effect remained stable while the trial-wise effect declined. Analysis of first-order sequential effects involved a subset of the data where, on lag -1 trials, there were no timeouts, and responses were correct without being "fast guesses" $(\mathrm{RT}<0.2 \mathrm{~s})$. First-order facilitation by target repetition was observed for correct RTs, which was greater when biasing was block-wise (0.042s for older participants, and $0.031 \mathrm{~s}$ for younger participants) than trial-wise $(0.025 \mathrm{~s}$ for older participants, and $0.005 \mathrm{~s}$ for younger participants). Facilitation by repetition was greater on cue-congruent (high-probability) than cue-incongruent targets, especially under block-wise biasing, and was modulated by age in that younger participants produced weaker effects, with negligible facilitation by repetition under trial-wise bias, including for cue-congruent targets. 


\section{LBA Parameter Estimation}

LBA parameter values were estimated by a hierarchical Bayesian procedure using a Markov Chain Monte Carlo sampler to generate posterior parameter distributions, as implemented in the Dynamic Models of Choice R library (https://osf.io/pbwx8/; Heathcote et al., 2018). Priors were assumed to be normal and diffuse (means of 2 and standard deviations of 3 for all of $A, B$ and $v, 1$ and 3 for $s v$, and $0.2 \mathrm{~s}$ and $0.25 \mathrm{~s}$ for $t 0$ ) with priors for $A, B$ and $s v$ having a lower bound of 0 , and $t_{0}$ having a lower bound of $0.1 \mathrm{~s}$ and an upper bound of $1 \mathrm{~s}$. Posterior distributions were estimated in multiple chains $(3 \times$ the number of model parameters), initially by separate estimation for each participant. These fits provided startpoints for separate hierarchical models for the older and younger groups that produced the final estimates. The hierarchical models assumed independent normal population distributions for each parameter with means with the same priors as used in individual fitting and standard deviations with exponential priors with a mean of 1 . Hierarchical sampling continued until convergence as assessed by visual inspection of trace plots and an $\widehat{R}$ statistic less than 1.1 (cf. Heathcote et al., 2018) in a final run of 250 iterations for each chain thinned by a factor of 10 . The scripts as well as trace plots and other output have been included in the OSF project associated with this manuscript. 


\section{LBA Model Comparison}

Model comparison was informed by the deviance information criterion (DIC; Spiegelhalter, Best, Carlin, \& van der Linde, 2014), a complexity-penalizing likelihood statistic for which smaller positive values, and larger negative values, by at least about 10 units indicate, by convention, better complexity-independent fits (cf. Heathcote et al., 2018). The bias-on-threshold-only model was very strongly preferred relative to the bias-on-rateonly model, by 290 and 655 units for older and younger participant datasets, respectively. The bias-on-rate-only model, although showing appropriate signs of biasing effects, failed to predict bias effects in the fastest RTs, mainly by under-estimating correct RTs for incongruent trials. DIC supported the modelling of bias effects on both thresholds and rates over the bias-on-threshold-only model by a smaller margin, 40 and 95 units for older and younger datasets, respectively. However, this model produced implausible $v$ estimates: they were higher for cue-incongruent than cue-congruent trials under all conditions, suggesting over-fitting. Hence, for further analyses we selected models in which bias only affected thresholds.

Estimating the effect of bias-type on $A$ led to an improvement of 51 and 22 DIC units for older and younger participant datasets, respectively. Further allowing a bias-type effect on to marginally improved this model by 13 and 3 DIC units respectively. Including estimation of $v$ per bias-type made no difference to the DICs for the younger dataset (reduced by 2 units) but substantially improved estimation for the older dataset (by 155 units). Accordingly, the final model included bias-type on all three of these parameters. Other models we considered were clearly excluded by much poorer DICs and/or qualitative failures to fit the data. The main alternative models and their fit measures are presented in Table 2. 
Table 3 presents root mean squared deviations (RMSDs) for the goodness-of-fit of model-predicted to the observed data presented in Figures 3 and 4 of the main report. The table also presents the observed range-normalized and observed mean-relative RMSD measures, and the normalized mean absolute error. The RMSDs were no more than about 50 ms for RTs and 20\% for ERs, which represents an appreciable goodness-of-fit in the context of the small degrees of freedom in this analysis. The fits can also be noted from these values to have been generally better for the older than younger participant data, and consistently so when accounting for the variability in the observations themselves. 
Table 2. Fit Measures per Alternative LBA Model Specifications.

\begin{tabular}{|c|c|c|c|c|c|c|c|c|c|}
\hline \multirow[b]{3}{*}{$N$} & \multicolumn{5}{|c|}{ LBA Parameters } & \multicolumn{4}{|c|}{ Group } \\
\hline & \multirow[b]{2}{*}{ A } & \multirow[b]{2}{*}{$B$} & \multirow[t]{2}{*}{ 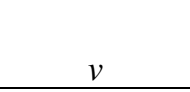 } & \multirow[b]{2}{*}{ sv } & \multirow[b]{2}{*}{$t_{0}$} & \multicolumn{2}{|c|}{ Older } & \multicolumn{2}{|c|}{ Younger } \\
\hline & & & & & & Min. Deviance & DIC & Min. Deviance & DIC \\
\hline 19 & 1 & B-C-R & B-D-M & $\mathrm{M}$ & 1 & 3376.0 & 4059.0 & -11042.8 & -10359.2 \\
\hline 20 & 1 & B-C-R & B-D-M & M & $\mathrm{B}$ & 3345.9 & 4034.8 & -11052.0 & -10354.8 \\
\hline 39 & 1 & B-R & B-D-C-T-M & M & 1 & 3126.5 & 4349.4 & -10893.0 & -9703.7 \\
\hline 43 & 1 & B-C-R & B-D-C-T-M & M & 1 & 2697.8 & 4019.3 & -11720.8 & -10454.0 \\
\hline 44 & 1 & B-C-R & B-D-C-T-M & M & $\mathrm{B}$ & 2662.8 & 3989.3 & -11723.6 & -10472.7 \\
\hline 20 & B & B-C-R & B-D-M & M & 1 & 3325.2 & 4008.3 & -11049.3 & -10381.3 \\
\hline 21 & $\mathrm{~B}$ & B-C-R & B-D-M & M & $\mathrm{B}$ & 3304.8 & 3995.3 & -11065.3 & -10383.8 \\
\hline
\end{tabular}

Note. $N=$ number of free parameters; all models included a constant value of for one level of sv.false. See text for definition of parameter and measure abbreviations in the column headings. For the conditions abbreviated under each parameter: $\mathrm{B}=$ bias-type; $\mathrm{C}=$ cue colour; $\mathrm{T}=$ target colour; $\mathrm{D}=$ discrimination difficulty; $\mathrm{R}=$ response (accumulator); $\mathrm{M}=$ match (of accumulator and response); 1 = intercept-only estimate. The model defined in the bottom row is the model that was selected for parameter estimates and contrasts, and optimality analysis.

Table 3. Goodness-of-fit of Observed and LBA-Predicted RTs and ERs for the Bias-onThreshold-Only Model.

\begin{tabular}{|c|c|c|c|c|c|c|}
\hline & \multicolumn{2}{|c|}{ Correct RT quantiles } & \multicolumn{2}{|c|}{ Error RT quantiles } & \multicolumn{2}{|c|}{ ER } \\
\hline & Older & Younger & Older & Younger & Older & Younger \\
\hline RMSD & 0.018 & 0.023 & 0.052 & 0.038 & 0.013 & 0.018 \\
\hline RMSD/range & 0.028 & 0.057 & 0.068 & 0.080 & 0.050 & 0.076 \\
\hline RMSD/mean & 0.022 & 0.038 & 0.056 & 0.065 & 0.085 & 0.101 \\
\hline MAE/sum-of-squares & 0.008 & 0.031 & 0.044 & 0.070 & 0.023 & 0.048 \\
\hline
\end{tabular}




\section{LBA Parameter Tests}

To capture the correlations between performance within each participant, all test results and intervals were obtained by firstly calculating the relevant condition difference for each participant then averaging these over participants for each iteration in each chain. Results for the threshold parameter $B$ are presented in the main report.

Accumulation rates. Table 4 presents estimates of mean rates $(v)$ per age-group and block-wise and trial-wise bias. Under block-wise biasing, older participants had larger matching rates than the younger participants for both difficulty levels, but younger participants had larger mismatching rates than older participants for easy trials, and there was no substantial difference between the age-groups for mismatching rates on hard trials. Under trial-wise biasing, older participants had larger matching and mismatching rates than the younger adults for both hard and easy trials. The difference between matching and mismatching rates (an index of the quality of stimulus evaluation) was larger for older than younger participants for both easy and hard trials, and whether biasing was block-wise $[d=$ $0.66(0.54,0.79), p<.001$ for easy trials, and $d=0.27(0.19,0.35), p<.001$ for hard trials] or trial-wise $[d=0.36(0.24,0.48), p<.001$ for easy trials, $d=0.12(0.04,0.20), p=.002$ for hard trials]. As expected, the difference between matching and mismatching rates was greater for the easy than hard trials in all cases (all $p \mathrm{~s}<.001$ ). Furthermore, the difficulty manipulation had a larger effect for older than younger participants under both block-wise biasing $[0.24(0.10,0.38), p<.001]$ and trial-wise biasing $[0.40(0.25,0.55), p<.001]$. 
Table 4. LBA Mean Accumulation Rate (v) Posterior Parameter Estimates: Medians of the mean over participants (and 95\% credible intervals).

\begin{tabular}{cllccc}
\hline Bias-type & Accumulator & Difficulty & Older & Younger & Older - Younger \\
\hline Block-wise & Match & Easy & $2.61(2.57,2.66)$ & $2.25(2.21,2.27)$ & $0.37(0.32,0.42), p<.001$ \\
& & Hard & $2.29(2.26,2.33)$ & $2.04(2.01,2.07)$ & $0.26(0.21,0.30), p<.001$ \\
\cline { 2 - 6 } & Mismatch & Easy & $0.37(0.26,0.48)$ & $0.66(0.59,0.73)$ & $-0.29(-0.43,-0.17), p<.001$ \\
& & Hard & $1.02(0.95,1.08)$ & $1.03(0.97,1.09)$ & $-0.01(-0.10,0.08), p=.401$ \\
\hline Trial-wise & \multirow{2}{*}{ Match } & Easy & $2.72(2.67,2.77)$ & $2.18(2.16,2.20)$ & $0.53(0.48,0.59), p<.001$ \\
& & Hard & $2.34(2.30,2.38)$ & $1.97(1.96,1.99)$ & $0.36(0.32,0.40), p<.001$ \\
\cline { 2 - 6 } & \multirow{2}{*}{ Mismatch } & Easy & $0.60(0.50,0.70)$ & $0.43(0.35,0.50)$ & $0.18(0.05,0.30), p=.003$ \\
& & Hard & $1.14(1.07,1.21)$ & $0.90(0.84,0.95)$ & $0.24(0.16,0.33), p<.001$ \\
\hline
\end{tabular}

The standard deviation of the rate parameter for the matching accumulator, $s v_{\text {Match, }}$ was larger for older $[0.700(0.685,0.716)]$ than younger $[0.516(0.506,0.527)]$ participants $[d$ $=0.183(0.165,0.202), p<.001]$. As the probability of making an error is a function of rate variability as well as the difference between matching and matching means rates, it is useful to calculate a quantity analogous to the signal-detection theory sensitivity measure:

$$
d^{\prime}=\left(v_{\text {Match }}-v_{\text {Mismatch }}\right) / \sqrt{s v_{\text {Match }}^{2}+s v_{\text {Mismatch }}^{2}}
$$

Under block-wise biasing, sensitivity was better for the older participants both easy $[0.43(0.33,0.54), p<.001]$ and hard $[0.15(0.08,0.22), p<.001]$ trials. Under trial-wise biasing, sensitivity was better for older than younger participants on easy $[0.17(0.07,0.28), p$ $<.001]$ but not hard $[0.02(-0.5,0.09), p=.256]$ trials. Sensitivity was always better for easy than hard trials (all $p s<.001$ ), and the sensitivity advantage for easy trials was larger for older than younger participants [block-wise: $0.29(0.16,0.41), p<.001$; trial-wise: 0.15 $(0.03,0.27), p=.006]$.

Startpoint noise (A). As shown in Table 5, A was larger for older than younger participants under both bias-types. For older participants, startpoint noise was larger under trial-wise than block-wise biasing $[d=0.188(0.110,0.267), p<.001]$. Conversely, for 
younger participants, startpoint noise was larger under block-wise than trial-wise biasing $[d=$ $0.085(0.046,0.126), p<.001]$.

Non-decision time (to). As also shown in Table 5, LBA-estimated non-decision time was longer for older than younger participants within each bias-type. For the differences between bias-types per age-group, older participants' non-decision time was longer under block-wise than trial-wise biasing $[d=0.021(0.006,0.035), p=.002]$ whereas younger participants' non-decision time was slightly longer under trial-wise than block-wise biasing $[d=0.009(0.001,0.017), p=.011]$.

Table 5. LBA Startpoint Variability $(A)$ and Non-decision Time $\left(t_{0}\right)$ Posterior Parameter Estimates: Medians of means over participants (and 95\% credible intervals).

\begin{tabular}{clccc}
\hline Parameter & Bias-type & Older & Younger & Older - Younger \\
\hline$A$ & Block-wise & $0.785(0.733,0.836)$ & $0.580(0.551,0.610)$ & $0.205(0.146,0.263), p<.001$ \\
& Trial-wise & $0.974(0.909,1.036)$ & $0.495(0.465,0.524)$ & $0.479(0.407,0.547), p<.001$ \\
\multirow{2}{*}{$t_{0}$} & Block-wise & $0.192(0.182,0.202)$ & $0.128(0.123,0.134)$ & $0.064(0.053,0.075), p<.001$ \\
& Trial-wise & $0.171(0.160,0.183)$ & $0.137(0.131,0.143)$ & $0.035(0.022,0.047), p<.001$ \\
\hline
\end{tabular}




\section{LBA Optimality Analysis}

We used simulation methods to determine the level of bias, defined in the proportional sense of Equation 1, which maximized accuracy conditional on the estimated non-threshold parameters and maintaining caution (i.e., average $B$ ) at the observed level. To illustrate, the relationship between bias and accuracy conditional on the average parameter estimates over all participants and conditions (see Table 6, final row) is shown in Figure 1a (simulation methods are described in the Figure caption). Figure $1 \mathrm{~b}$ plots of estimated optimal bias as a function of the average $B$ value for four base-rate odds ratios. It shows that optimality requires more extreme biases at lower levels of caution (i.e., average $B$ ). Figure 1c shows that their asymptotic values are an approximately linear function of the logarithm of the odds ratios, similar to the exact linear relationship derived by Bogacz, Brown, Moehlis, Holmes, and Cohen (2006) for the "simple" DDM (i.e., without trial-to-trial parameter variability that is usually assumed in order to capture benchmark phenomena).

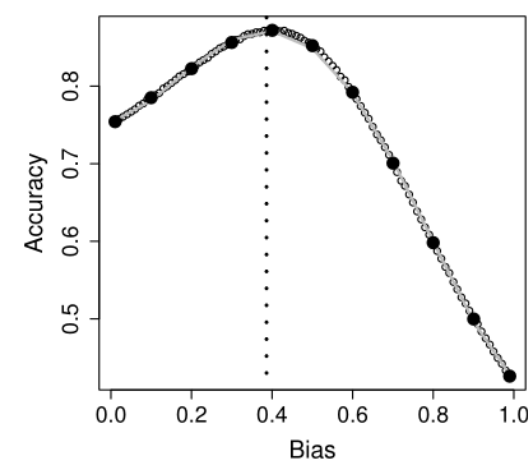

(a)

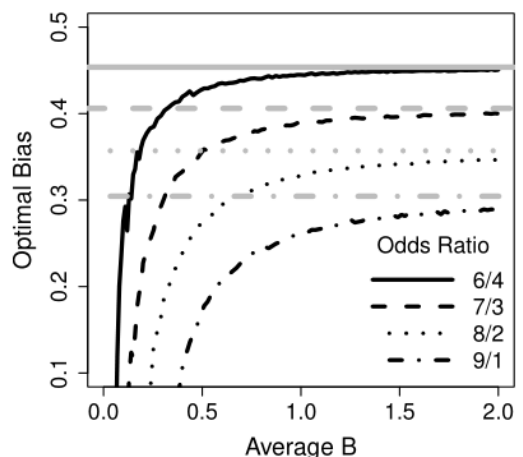

(b)

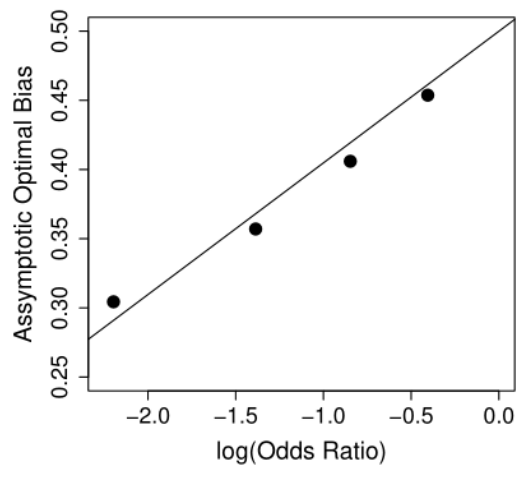

(c)

Figure 1. Accuracy by Bias, and Optimal-bias by Caution and Log-Odds Ratio. Panel (a) plots accuracy as a function of 99 bias values (open circles, using 100,000 simulations each for the two stimuli) based on the mean over base-rate conditions and groups (see Table 6) of posterior parameter estimates, and fitted $4^{\text {th }}$-order polynomial (grey line) based on the set of 
11 simulated values (based on 200,000 simulated trials each) indicated by solid points (with corresponding bias $0.01,0.1,0.2, \ldots 0.9,0.99)$. The optimum bias estimate is given by the peak of the fitting function (indicated by a dotted vertical line at a value of 0.389 ), which was determined from the root of its derivative of the polynomial corresponding to the highest point on the interval between the 11 values. Panel (b) plots estimated optimal bias as a function of the average $B$ value for different stimulus one vs. two odds ratios (dark lines) and horizontal lines showing estimated asymptotic bias (based on 2,000,000 simulated trials). Panel (c) plots asymptotic bias for the four curves in (b) as a function of the logarithm of the odds ratio for each.

Table 6. LBA Mean Parameter Estimates for per Age Group and Bias-type.

\begin{tabular}{llccccccccc}
\hline Age-Group & Bias-type & $A$ & $B_{\mathrm{CC}}$ & $B_{\mathrm{CI}}$ & $v_{\mathrm{E}-\mathrm{M}}$ & $v_{\mathrm{H}-\mathrm{M}}$ & $v_{\mathrm{E}-\mathrm{MM}}$ & $v_{\mathrm{H}-\mathrm{MM}}$ & $s v_{\mathrm{MM}}$ & $t_{0}$ \\
\hline Older & Block-wise & 0.79 & 0.96 & 1.22 & 2.61 & 2.29 & 0.37 & 1.02 & 0.70 & 0.19 \\
& Trial-wise & 0.97 & 1.09 & 1.24 & 2.72 & 2.34 & 0.60 & 1.14 & 0.70 & 0.17 \\
Younger & Block-wise & 0.58 & 0.62 & 0.79 & 2.24 & 2.04 & 0.66 & 1.03 & 0.52 & 0.13 \\
& Trial-wise & 0.49 & 0.67 & 0.75 & 2.18 & 1.97 & 0.43 & 0.89 & 0.52 & 0.14 \\
\hline Average & & 0.71 & 0.84 & 1.00 & 2.44 & 2.16 & 0.51 & 1.02 & 0.61 & 0.16 \\
\hline
\end{tabular}

Note. $s v_{\text {Mm }}$ was fixed at 1 . Subscripts for parameters are: $\mathrm{CC}=$ cue-congruent, $\mathrm{CI}=$ cueincongruent, $\mathrm{E}=$ easy stimulus, $\mathrm{H}=$ hard stimulus, $\mathrm{M}=$ matching accumulator, $\mathrm{MM}=$ mismatching accumulator.

We investigated how each non-threshold parameter affected bias curves in order to determine how they affected the optimality advantage for older participants. For the odds ratio used in the experiments (7/3), Figure 2 explores the effects on bias functions (as in Figure $1 b$ ) of manipulating each non-threshold parameter while keeping the others fixed. The average parameter vector was used as a baseline and the manipulation aimed to vary the 
targeted parameter over the range of all values shown in Table 6. Figure 2a shows that asymptotic optimal bias is virtually unaffected by $A$. However, the rate of increase toward asymptote depends strongly on $A$, slowing as startpoint noise increases. As $A$ increases there is a lower bound on the average $B$ below which the optimal bias is no longer greater than zero, hence the curves in Figure $2 \mathrm{a}$ shift to the right as well as approach their asymptote more slowly as $A$ increases. Figure $2 \mathrm{~b}$ shows that the other trial-by-trial variability parameter, $s v$, has a similar but much smaller effect on the rate of approach to asymptote and a larger, although still relatively small, effect on the asymptote. Figures $2 \mathrm{c}$ and $2 \mathrm{~d}$ show that both the average rate for the two accumulators and the difference between matching and mismatching accumulators have a large effect on the asymptote and the rate with which it is approached.

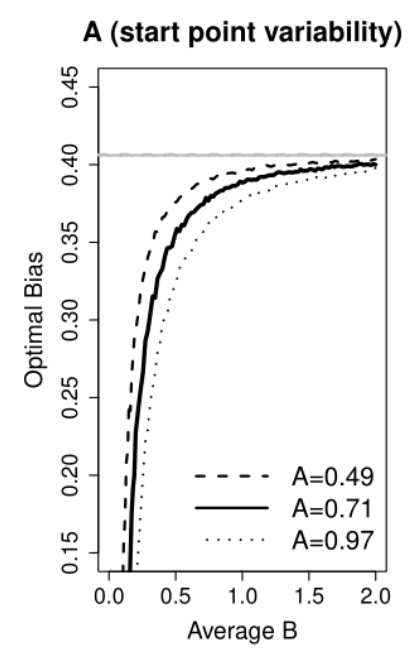

(a)

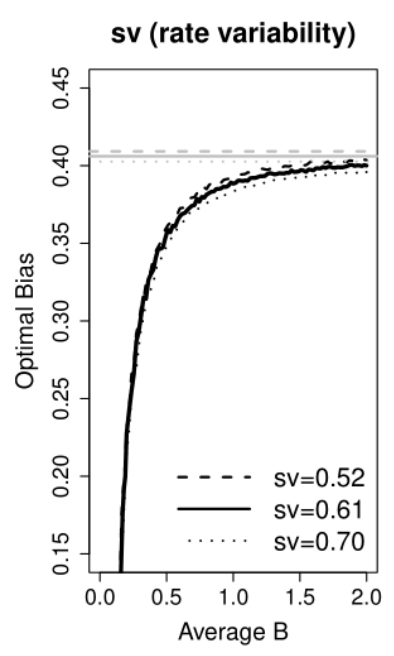

(b)

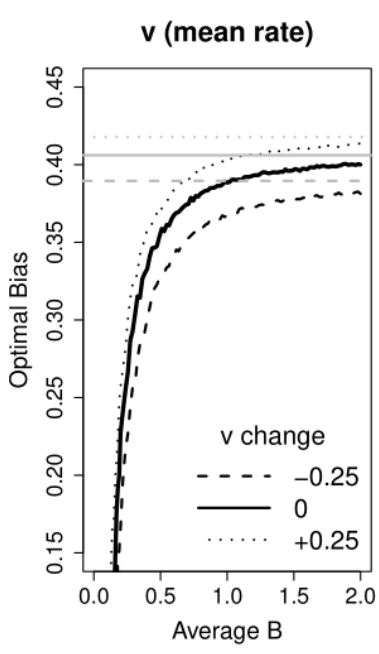

(c)

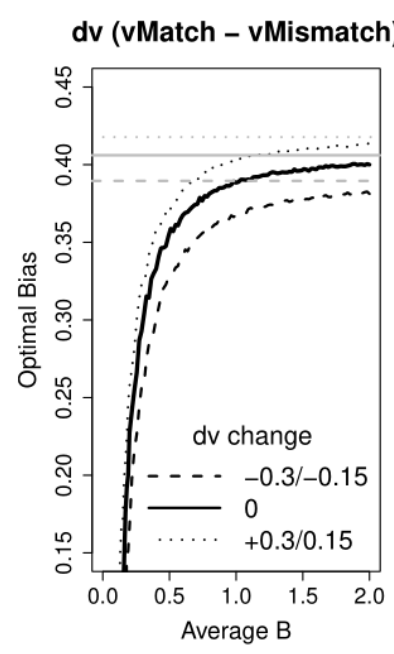

(d)

Figure 2. Optimal bias functions for the young trial vector in Table 6 (solid line) with varying values of (a) startpoint noise ( $A$ : values shown in legend), (b) mean rate $(v)$ for both accumulators (either adding or subtracting 0.2 from both), (c) rate standard deviation ( $s v$ ) for the matching accumulator (adding either 0.2 or 0.4 ) and (d) the difference between mean rates for matching and mismatching accumulators (with difference symmetrically expanded or contracted around the baseline value for the young trial vector). 


\section{Diffusion Decision Model}

We fit two DDMs, corresponding to the best LBA model where bias only affected threshold parameters (top row, Table 7) and one where bias only affected the mean rate of accumulation (bottom row, Table 7). In the former model allowing the cue colour factor to affect both the DDM $a$ and $z$ parameters corresponds to allowing different thresholds for each accumulator as a function of cue colour in the LBA. In the latter model allowing cue-colour to affect the DDM $v$ parameter corresponds to allowing the same effect on the LBA $v$ parameter. As in the final model selected for the LBA analysis, these models also estimated effects of bias-type on startpoint noise and non-decision time.

Table 7. Fit Measures per Alternative DDM Specifications.

\begin{tabular}{|c|c|c|c|c|c|c|c|c|c|}
\hline \multicolumn{6}{|c|}{ DDM Parameters } & \multicolumn{4}{|c|}{ Group } \\
\hline \multirow[b]{2}{*}{$N$} & \multirow[b]{2}{*}{$a$} & \multirow[b]{2}{*}{$z$} & \multirow[b]{2}{*}{$v$} & \multirow[b]{2}{*}{$s v$} & \multirow[b]{2}{*}{$t_{0}$} & \multicolumn{2}{|l|}{ Older } & \multicolumn{2}{|l|}{ Younger } \\
\hline & & & & & & Min. Deviance & DIC & Min. Deviance & DIC \\
\hline 18 & $\mathrm{~B}-\mathrm{C}$ & $\mathrm{B}-\mathrm{C}$ & B-D & 1 & B & 3568.7 & 4175.3 & -10481.2 & -9870.3 \\
\hline 28 & B & B & B-D-C-T & 1 & B & 3443.0 & 4366.0 & -10264.9 & -9509.8 \\
\hline
\end{tabular}

Note. $N=$ number of free parameters. See text for definition of parameter and measure abbreviations in the column headings. For the conditions abbreviated under each parameter: $\mathrm{B}=$ bias-type $\mathrm{C}=$ cue colour; $\mathrm{T}=$ target colour; $\mathrm{D}=$ discrimination difficulty $; 1=$ interceptonly estimate. The model defined in the top row of values is the model that was selected for parameter estimates and contrasts, and optimality analysis.

As can be seen from the DIC values listed in Table 7, the model in which bias only affected threshold parameters (top row) provided a better fit to the data than the model in which bias affected only the mean rate of accumulation (bottom row), corresponding to the results for comparable LBA models represented in Table 2. Comparing the DIC values for 
the bias-on-threshold-only models between the LBA and the DDM, it can be seen (with respect to the complexity-penalizing attributes of the DIC) that the LBA provided a better account of the data of both older and younger participants (with reductions by 180 and 514 DIC units, respectively).

We continue with parameter tests and optimality analyses of the best DDM parameterization; see the main report for description of the comparison tests, and above for description of the optimality analysis.

Caution (a). As can be seen from the data presented in Table 8, DDM-estimated caution was larger among older than younger participants under both bias-types. This replicates the common result in the literature, and the present result for LBA-estimated caution. Comparing estimates between bias-types per age-group, caution was slightly lower under block-wise than trial-wise bias among older participants, with no such difference among younger participants, $[d=-0.06(-0.09,-0.03), p<.001$, for older participants, and $d$ $=0.01(-0.01,0.03), p=.279$, for younger participants], which also fully replicates the finding for LBA average thresholds.

Table 8. DDM Caution (a) Posterior Parameter Estimates: Medians of means over cue colour and participants (and 95\% credible intervals).

\begin{tabular}{lccc}
\hline \multicolumn{1}{c}{ Bias-type } & Older & Younger & Older - Younger \\
\hline Block-wise & $1.33(1.31,1.35)$ & $0.99(0.97,1.00)$ & $0.34(0.32,0.37), p<.001$ \\
Trial-wise & $1.39(1.36,1.41)$ & $0.98(0.96,1.00)$ & $0.41(0.38,0.43), p<.001$ \\
\hline
\end{tabular}

Response bias (z/a). Table 9 presents descriptives for the startpoints $(z)$ relative to boundary separation ( $a$ ) averaged over cue colours such that positive deviations from the neutral startpoint of $z / a=0.5$ represent a bias in startpoint toward the cue-congruent 
boundary. As these descriptives indicate, and as comparison tests confirm, these deviations were substantial under block-wise biasing for both older $[d=0.100(0.093,0.108), p<.001]$ and younger $[d=0.095(0.087,0.103), p<.001]$ participants, and under trial-wise biasing, again for both older $[d=0.045(0.038,0.053), p<.001]$ and younger $[d=0.049(0.042$, $0.057), p<.001]$ participants. This demonstrates the expected reduction of startpoint towards the response boundary that was congruent with the colour cued as most likely to be the target. Age-related differences in the effect of cue-congruence are presented in the right-most column of Table 9, from which it can be seen that the block-wise biasing effect was slightly greater among older than younger participants. This replicates the same but more substantial age-difference seen by the LBA-estimated effect of cue-congruence. Conversely, the trialwise biasing effect tended to be larger among younger than older participants (opposite to what was seen with the LBA-estimated effect), but this difference was small and unreliable. Between bias-types per age, the shift in startpoint from 0.5 was larger under block-wise than trial-wise biasing for both older $[d=0.055(0.044,0.066), p<.001]$ and younger $[d=0.046$ $(0.035,0.056), p<.001]$ participants, with this bias-type difference in the congruence effect being larger among the older relative to the younger participants but-as with the LBA estimates—not to a substantial or reliable extent $[d=0.010(-0.005,0.025), p=.099]$.

Table 9. DDM Relative Startpoint (z/a) Posterior Parameter Estimates: Medians of means over participants (and 95\% credible intervals).

\begin{tabular}{lccc}
\hline Bias-type & Older & Younger & Older - Younger \\
\hline Block-wise & $0.600(0.593,0.608)$ & $0.595(0.587,0.603)$ & $0.005(-0.005,0.016), p=.158$ \\
Trial-wise & $0.545(0.538,0.553)$ & $0.549(0.542,0.557)$ & $-0.004(-0.015,0.007), p=.222$ \\
\hline
\end{tabular}

Drift-rate (v). With responses boundaries categorised as to response category rather than accuracy, there is no matching versus mismatching drift rate for this instantiation of the 
DDM such as there was for the corresponding LBA model. An analysis of drift rate yet corresponds to the matching minus mismatching difference in rates that was computed for the LBA. As shown in Table 10, and as expected, rates were faster for easy than hard trials. Agerelated effects differed per bias-type. Under block-wise biasing, older participants had larger drift rates relative to younger participants under both difficulty levels, most consistently on easy trials. This replicates the results for the LBA. However, under trial-wise biasing, younger participants had larger rates than older participants, most consistently on hard trials, which was never indicated under any analysis of the LBA estimates and is inconsistent with the performance data.

Table 10. DDM Mean Drift Rate (v) Posterior Parameter Estimates: Medians of means over participants (and 95\% credible intervals).

\begin{tabular}{clccc}
\hline Bias-type & Difficulty & Older & Younger & Older - Younger \\
\hline Block-wise & Easy & $2.78(2.68,2.88)$ & $2.50(2.40,2.60)$ & $0.28(0.13,0.41), p<.001$ \\
& Hard & $1.53(1.46,1.60)$ & $1.43(1.35,1.51)$ & $0.10(0.00,0.20), p=.027$ \\
Trial-wise & Easy & $2.71(2.61,2.82)$ & $2.86(2.75,2.97)$ & $-0.15(-0.30,0.01), p=.031$ \\
& Hard & $1.37(1.30,1.45)$ & $1.52(1.44,1.60)$ & $-0.15(-0.26,-0.04), p=.006$ \\
\hline
\end{tabular}

Startpoint noise (sz). As shown in Table 11, DDM-estimated startpoint noise was slightly less for older than younger participants under both bias-types, but these age differences were not compelling. The direction of the age difference was opposite to the corresponding but more substantial and reliable result seen in the LBA estimates. Startpoint noise was larger under trial-wise than block-wise biasing for both older $[d=0.026(-0.041$, $0.094), p=.228]$ and younger $[d=0.036(-0.040,0.109), p=.179]$ participants. This replicates the bias-type differences seen for older but not younger participants for the like parameter $(A)$ in the LBA, but again with differences that were not as compelling as those seen with the LBA. 
Non-decision time (to). As also shown in Table 11, DDM-estimated non-decision time was longer for older than younger participants within each bias-type. For the differences between bias-types per age-group, older participants' non-decision time was longer under block-wise than trial-wise biasing $[d=0.012(0.007,0.017), p<.001]$ whereas younger participants' non-decision time was longer under trial-wise than block-wise biasing $[d=$ $0.015(0.012,0.019), p<.001]$. This replicates the results for the LBA estimates except that the difference in non-decision time between bias-types was larger by the LBA than by the DDM for older participants, but larger by the DDM than by the LBA for younger participants, with the result that the bias-type difference among younger participants was more compelling by the DDM than by the LBA. The DDM point estimates for minimum nondecision times, including of the difference between older and younger participants per biastype, were about twice as large, and the credible intervals were about half the range, as compared with the corresponding LBA estimates.

Table 11. DDM Startpoint Variability $(s z)$ and Non-decision Time $\left(t_{0}\right)$ Posterior Parameter Estimates: Medians of means over participants (and 95\% credible intervals).

\begin{tabular}{clccl}
\hline Parameter & Bias-type & Older & Younger & Older - Younger \\
\hline \multirow{2}{*}{$s z$} & Block-wise & $0.257(0.211,0.305)$ & $0.272(0.220,0.323)$ & $-0.015(-0.083,0.056), p=.340$ \\
& Trial-wise & $0.283(0.233,0.334)$ & $0.308(0.253,0.362)$ & $-0.024(-0.095,0.051), p=.264$ \\
\multirow{2}{*}{$t_{0}$} & Block-wise & $0.399(0.395,0.402)$ & $0.280(0.278,0.282)$ & $0.118(0.114,0.122), p<.001$ \\
& Trial-wise & $0.387(0.383,0.390)$ & $0.296(0.293,0.298)$ & $0.091(0.086,0.095), p<.001$ \\
\hline
\end{tabular}

Optimality analyses. As can be noted from the data presented in Table 12, participants set their response bias closer to the optimal level under block-wise than trial-wise biasing. The values in this table and the following graph correspond to the mean estimates of the DDM parameters. We now test differences between observed and optimal bias as a function 
of the DDM $z$ parameter by averaging over optimality estimates for each posterior parameter sample. This leads to slightly different mean values of the difference compared to the Table 12 "Bias Difference" column. As was the case for the LBA, the departure from optimality was smaller for older than younger participants under block-wise biasing [older: 0.014 $(-0.001,0.036)$; younger: $0.028(0.009,0.046), d=0.014(-0.012,0.039), p=.836]$, but it was larger for older than younger participants under trial-wise biasing [older: $0.075(0.052$, 0.094); younger: $0.064(0.045,0.081) ; d=-0.011(-0.037,0.019), p=.202]$. Further, uncertainty about these differences was larger than for the LBA, so that the Bayesian $p$ values in each case did not indicate strong evidence. The predicted benefit to accuracy by setting an optimal bias was slightly larger than in the LBA model predictions. For both groups, the observed bias was closer to optimal under block-wise than trial-wise biasing [older: $0.06(0.03,0.09), p<.001$; younger: $0.03(0.01,0.06), p=.010]$.

Table 12. DDM Observed (OBS) and Optimal (OPT) Accuracy (\%C), and Observed and Optimal Proportional Bias. Note that bias vales less than 0.5 favour the cue-congruent response.

\begin{tabular}{llccccc}
\hline Age Group & Bias-Type & $\% \mathrm{C}_{\mathrm{OBS}}$ & $\%_{\mathrm{CPT}}$ & Bias $_{\mathrm{OBS}}$ & Bias $_{\mathrm{OPT}}$ & Bias Difference \\
\hline Older & Block-wise & 86.8 & 89.4 & 0.401 & 0.384 & 0.017 \\
& Trial-wise & 86.0 & 88.5 & 0.455 & 0.379 & 0.076 \\
Younger & Block-wise & 81.5 & 86.0 & 0.406 & 0.380 & 0.026 \\
& Trial-wise & 83.9 & 87.7 & 0.452 & 0.385 & 0.067 \\
\hline
\end{tabular}



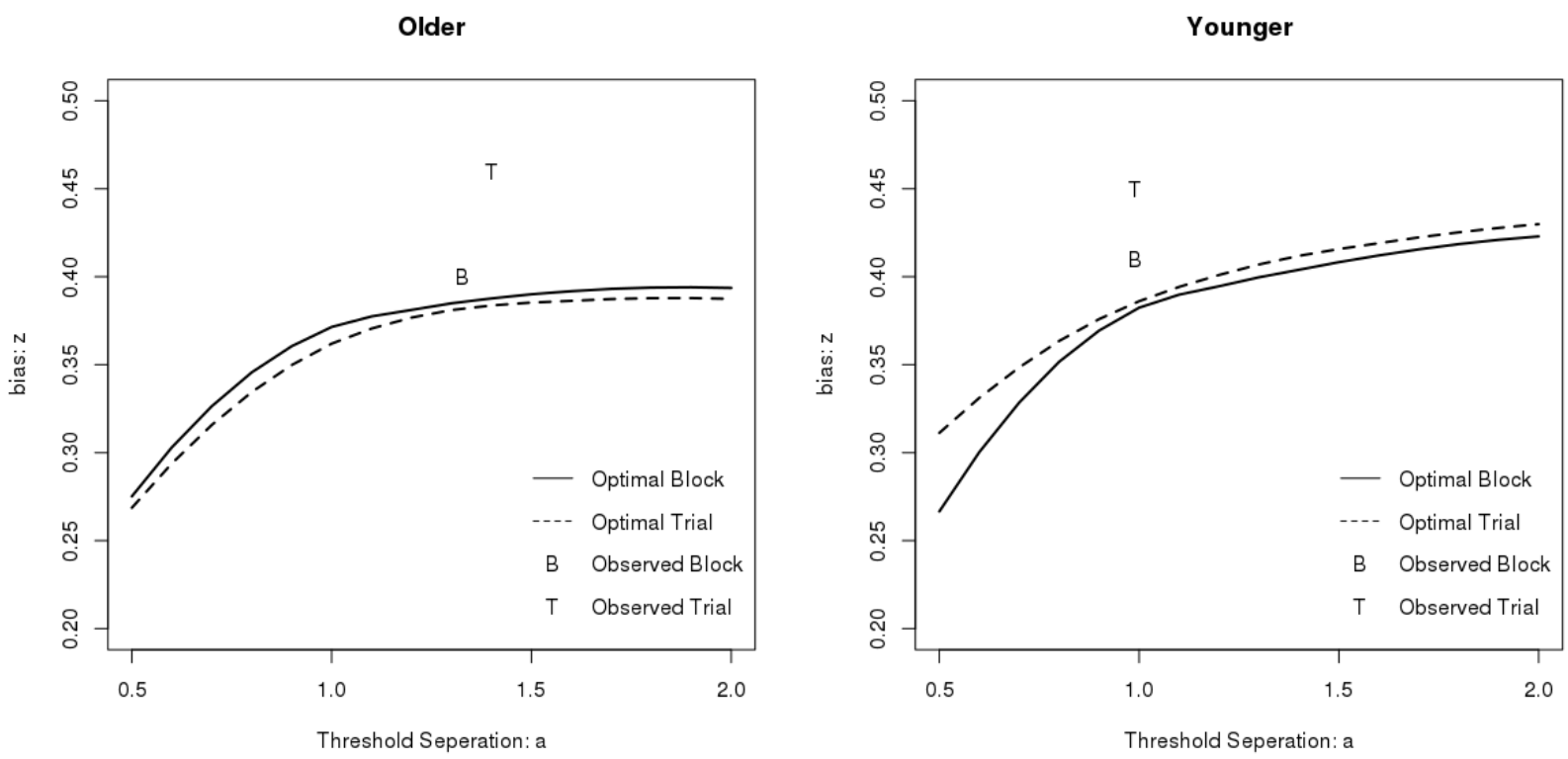

Figure 3. Optimal bias curves and observed block-wise (B) and trial-wise (T) bias per age group. Note that DDM tended toward ceiling as threshold separation increased, making calculation of an asymptotic bias computationally challenging, and so these values are not included on the graph. 


\section{Supplemental References}

Baayen, R. H., Davidson, D. J., \& Bates, D. M. (2008). Mixed-effects modeling with crossed random effects for subjects and items. Journal of Memory and Language, 59, 390412. http://doi.org/10.1016/j.jml.2007.12.005

Baayen, R. H., \& Milin, P. (2010). Analyzing reaction times. International Journal of Psychological Research, 3, 12-28. http://doi.org/10.21500/20112084.807

Bartoń, K. (2018). MuMIn: Multi-model inference (Version 1.42.1) [R package]. Retrieved from https://CRAN.R-project.org/package=MuMIn

Bates, D., Mächler, M., Bolker, B., \& Walker, S. (2015). Fitting linear mixed-effects models using lme4. Journal of Statistical Software, 67, 1-48.

http://doi.org/10.18637/jss.v067.i01

Bogacz, R., Brown, E., Moehlis, J., Holmes, P., \& Cohen, J. D. (2006). The physics of optimal decision making: A formal analysis of models of performance in twoalternative forced-choice tasks. Psychological Review, 113, 700-765. http://doi.org/10.1037/0033-295X.113.4.700

Davies, R. A. I., Arnell, R., Birchenough, J. M. H., Grimmond, D., \& Houlson, S. (2017). Reading through the life span: Individual differences in psycholinguistic effects. Journal of Experimental Psychology: Learning, Memory, and Cognition, 43, 12981338. http://doi.org/10.1037/xlm0000366

Eager, C., \& Roy, J. (2017). Mixed effects models are sometimes terrible. Retrieved from https://arxiv.org/abs/1701.04858

Heathcote, A., Lin, Y., Reynolds, A., Strickland, L., Gretton, M., \& Matzke, D. (2018). Dynamic models of choice. Behavior Research Methods. https://doi.org/10.3758/s13428-018-1067-y 
Kuznetsova, A., Brockhoff, P. B., \& Christensen, R. H. B. (2017). lmerTest: Tests in linear mixed effects models. Journal of Statistical Software, 82, 1-26. http://doi.org/10.18637/jss.v082.i13

Silverman, I. (1963). Age and the tendency to withhold response. Journal of Gerontology, 18, 372-375. http://doi.org/10.1093/geronj/18.4.372

Singmann, H., \& Kellen, D. (in press). An introduction to mixed models for experimental psychology. In D. H. Spieler \& E. Schumacher (Eds.), New methods in neuroscience and cognitive psychology: Psychology Press.

Spiegelhalter, D. J., Best, N. G., Carlin, B. P., \& van der Linde, A. (2014). The deviance information criterion: 12 years on. Journal of the Royal Statistical Society: Series B (Statistical Methodology), 76, 485-493. http://doi.org/10.1111/rssb.12062 